\title{
L'invraisemblable de la narration omnisciente
}

Suzette ALI, CRILCQ, Université de Québec à Montréal

Le point de vue omniscient permet au narrateur de posséder un savoir illimité sur les événements qu'il rapporte et, le plus souvent, il lui accorde un pouvoir absolu sur le déroulement des actions. Cependant, l'ambiguité qui entoure cette technique narrative, pousse beaucoup de romanciers contemporains à renouveler son utilisation dans les récits. Ces renouvellements s'écartent des conditions habituelles de son emploi dans la mesure où le narrateur omniscient devient incompétent. On voit ce dernier ignorer certains événements de l'histoire qu'il raconte, et maîtriser difficilement ses personnages. La déstabilisation de ce type de narration est très présente, par exemple, dans Dernier amour de Christian Gailly, La maison des temps rompus de Pascale Quiviger et Tarmac de Nicolas Dickner. Ces romans choisis pour notre étude ont la particularité d'introduire un narrateur homodiégétique omniscient. En principe, le narrateur à la première personne est un personnage identifié, donc non anonyme, et dépourvu d'omniscience. Son point de vue et son savoir sont conventionnellement limités. L'acquisition d'un savoir illimité par les narrateurs-personnages des romans étudiés dans cet article paraît dès lors problématique. Leurs connaissances dépassent la perception soumise aux contraintes reconnues de la narration homodiégétique. Toutefois, elles présentent plusieurs lacunes. Cela constitue une altération des conventions des deux types de narration et nécessite que l'on y réfléchisse.

\section{Narrateurs homodiégétiques omniscients}

Le premier des romans qui seront analysés, est Dernier amour de Gailly, publié en 2004 aux éditions de Minuit. Dans ce roman, la situation narrative à partir de laquelle l'histoire se raconte pose problème. Le texte commence, en effet, avec un point de vue objectif et omniscient, le narrateur étant au début du roman à l'extérieur des événements qu'il rapporte. Mais cette position du narrateur change au fur et à mesure qu'on avance dans la lecture de l'histoire. Celuici s'insère progressivement au sein du récit qu'il produit, provoquant de la sorte plusieurs transgressions des conventions de la narration. À cette extravagante situation narrative s'ajoute l'originalité de l'omniscience de celui-ci. Étant au début du roman extradiégétique et hétérodiégétique, le narrateur omniscient est capable de lire dans les pensées des différents 
personnages présents dans le roman, chose qui le distingue du personnage nommé Paul Cédrat dont le savoir est limité. Mais cette habileté du narrateur extradiégétique à tout savoir ne disparait pas lorsque ce même narrateur devient homodiégétique et adopte un statut identique à celui des autres personnages. Au contraire, son omniscience prétend même deviner les pensées des lecteurs. De plus, l'irrégularité de l'omniscience dans ce roman s'accentue lorsque la fusion de Paul Cédrat avec le narrateur entraîne une confusion et une fusion entre la perspective restreinte du personnage et la perspective omnisciente du narrateur et engendre une fluctuation du point de vue omniscient. Cela a pour conséquence de rendre incertain le savoir du narrateur. C'est dire que malgré son omniscience, le «je » n'arrive pas à tout savoir. La narration omnisciente se trouve ainsi déstabilisée dans le roman ou, tout au moins, largement problématisée.

Dans La maison des temps rompus de Quiviger paru en 2008, la narratrice reste non identifiée jusqu'à la fin de l'histoire. L'emploi du pronom personnel «je» ne permet pas de repérer l'identité de la personne qui parle. Malgré la présence de certains indices dans le texte nous indiquant qu'elle peut être soit Claire soit Lucie, l'une des deux protagonistes de l'histoire qui sera racontée, il est toujours difficile de déterminer laquelle des deux femmes prend en charge le récit à cause de la fusion entre ces deux personnes. Non seulement l'identité de la narratrice fait problème, mais aussi, son omniscience. Le fait de relater en profondeur diverses histoires de femmes qu'elle a à peine connues ou rencontrées, met en évidence la supériorité de son savoir. Son histoire contient également beaucoup d'anticipations et de digressions qui constituent, à leur tour, une preuve de son omniscience. Être omnisciente sert à cette narratrice à comprendre une histoire traumatisante qui est la mort d'une petite fille nommée Odyssée, de même que cela lui permet de contrôler le déroulement des actions de l'histoire. De fait, il y a une volonté de la part du «je » de tout savoir en même temps que de tout contrôler. Mais malgré la domination de la narratrice sur l'histoire, elle restera toujours incapable de sauver Odyssée de la mort. Son omniscience apparaît donc inefficace.

Tarmac est un roman que Dickner a publié en 2009. Dans ce roman, l'identité du «je » qui assume le récit, est moins problématique que celle des autres narrateurs cités plus haut. Malgré le fait que celle-ci n'est révélée que progressivement et que tous les autres personnages sont identifiés dès leur première apparition à l'exception du narrateur, le «je » assure la crédibilité de ses paroles. Les transgressions de la vraisemblance narrative ne commencent que 
lorsque ce narrateur homodiégétique acquiert un savoir supérieur sur des événements auxquels il n'a jamais participé. À partir du chapitre 49, on remarque un changement imprévu de la perspective narrative. Le point de vue interne du narrateur devient omniscient, ce qui lui permet de raconter les aventures de Hope à New York puis au Japon sans y avoir assisté. Plus encore, le narrateur avoue n'avoir jamais parlé à Hope depuis son départ vers le Japon, et cela après plusieurs chapitres de descriptions minutieuses de New York, de Tokyo et des périples de Hope dans ces différents endroits. Le savoir du narrateur dans ce roman pose donc problème. Tout d'abord, rien ne vient justifier dans le récit d'où le narrateur tient toutes ces informations sur le voyage de son amie. Ensuite, certaines scènes semblent inventées par lui, dans la mesure où l'imagination paraît la seule explication possible à ces connaissances profondes. Grâce à elle, le narrateur pourrait combler son manque d'informations sur l'histoire racontée.

Bref, plusieurs points communs rapprochent les romans étudiés. Les narrateurs homodiégétiques acquièrent tous une vision omnisciente du monde auquel ils appartiennent, à des étapes différentes de leur évolution dans ce monde. Par ailleurs, cette omniscience est altérée. La narration omnisciente est, en effet, utilisée dans ces romans de façon à faire ressortir son caractère invraisemblable, tel que nous le verrons dans ce qui suit.

\section{Les altérations du point de vue omniscient dans les romans étudiés}

La transgression des critères romanesques traditionnels, en d'autres termes l'omniscience irrégulière des narrateurs homodiégétiques, ne conduit pas à leur accorder une plus grande autorité ou un savoir absolu. Au contraire, elle transforme les informations que ceux-ci présentent en des données incertaines. Des fantasmes, des mensonges et d'autres inventions irréelles et fantastiques s'introduisent dans leurs histoires et déstabilisent le monde où se déroulent les actions. La transgression des normes de la narration omnisciente prend donc plusieurs formes dans les romans. Alors que le narrateur omniscient classique garantit un savoir complet des faits rapportés, même si cette compétence constitue un artifice de la narration, les narrateurs des romans étudiés sèment le doute quant à leur compétence mais aussi quant à la pertinence de cette convention narrative.

Les altérations de l'omniscience se manifestent de différentes manières dans chacun des romans. Dans La maison des temps rompus, ce sont les troubles progressifs de la vue de la narratrice qui nous signalent la présence des problèmes d'omniscience : «D'ici, je domine 
parfois le paysage, parfois je ne vois rien » (Quiviger 13). Dans Tarmac, ce sont les va-et-vient constants entre le point de vue interne restreint et le point de vue omniscient qui déstabilisent la consistance de ce dernier : «À quoi pouvait bien ressembler Tokyo à l'heure du déjeuner ? J'imaginais des gouttières, des flaques d'eau, un ciel pluvieux - la saison des typhons commençait dans quelques jours et on annonçait des précipitations abondantes pour plusieurs semaines. Merci, Canal Météo » (Dickner 207), affirme le narrateur après plusieurs chapitres d'omniscience et de descriptions minutieuses de Tokyo, du Japon et de l'histoire de ce pays selon le point de vue de ses habitants. Le narrateur retourne ici à un point de vue personnel et son savoir restreint, issu du Canal météo, nous induit à croire qu'il a rompu avec l'omniscience. Pourtant, ce n'est pas exactement ce qui arrive. Le narrateur redevient omniscient plus loin dans le récit et reprend la narration du reste de l'histoire de Hope en faisant valoir un savoir complet. Cela rend donc ambigus et la situation de narration et le point de vue omniscient dans ce roman. Dans Dernier amour, c'est la fusion et même la confusion entre Paul Cédrat et le narrateur qui détruit la stabilité de l'omniscience de ce dernier. La réunion de ces deux sujets entraîne, en effet, une fusion et dès lors une confusion entre point de vue interne et point de vue omniscient. Aucun de ces deux points de vue ne domine plus dans le roman et les fluctuations visuelles et cognitives ont pour effet de rendre plus lacunaire le savoir du narrateur : «Ils sont intrigués. Troublés peut-être. Oui, c'est possible. Ils se disent que cet homme, ce genre d'homme dans le passé devait. Oui, peut-être » (Gailly 63-64). Cette instabilité de 1'omniscience va en augmentant jusqu'à rendre invraisemblable la situation de narration : «Elle est entrée dans l'espace enfumé avec une peur au cœur. Cette peur n'avait rien à voir avec l'idée ou la vision de Paul mort seul dans la villa. Ou tout à voir. C'est plutôt ça. La peur de le revoir comme ça » (Gailly 117). On remarque, dans cette citation, que le narrateur a accès aux sentiments du personnage dont il reconnaît la peur. Cependant, cette omniscience n'est pas consistante à cause des hésitations qui la déstabilisent. Ces oscillations entre savoir illimité et savoir restreint rendent suspect le point de vue omniscient du narrateur et sa prétention à tout savoir.

Si les théoriciens et critiques contemporains, notamment Alain Rabatel et Pierre Vitoux, admettent dans leurs analyses la possibilité d'une omniscience discontinue dans un récit, ils expliquent cette discontinuité par le choix que peut effecteur le narrateur hétérodiégétique d'adhérer ou non au point de vue de son personnage : 
[...] le même narrateur, voire le même focalisateur [...], dans la même œuvre, est capable de varier les visions et les profondeurs de perspective, "tout naturellement", en fonction des référents, des situations, des intentions communicationnelles, etc., sans qu'il soit nécessaire de parler hors de propos d'altérations du principe de l'omniscience du narrateur, ou d'invoquer un brusque passage en focalisation externe... (Rabatel 102)

On ne peut, par contre, consentir à accorder ce choix aux narrateurs des trois romans puisqu'aucun des deux points de vue interne et omniscient n'y domine. Ces derniers ne font qu'alterner, plus précisément, s'infiltrer l'un dans l'autre jusqu'à créer la confusion des points de vue et la transgression de l'omniscience. La double position des narrateurs, qui, dans les propos de Rabatel, vient mettre en lumière la restriction du point de vue interne, met plutôt en doute dans nos romans l'efficacité de la narration omnisciente.

Cette dernière n'est pas la solution aux lacunes du point de vue personnel car, comme nous le remarquons dans ces romans, elle n'est pas utilisée pour tout connaitre et tout contrôler. Les narrateurs, à l'exception de la narratrice de La maison des temps rompus, avouent leur ignorance de beaucoup d'événements importants malgré leur omniscience. C'est le cas de Michel Bauermann dans Tarmac, qui annonce précisément ne pas être au courant de ce que vit son amie à l'étranger, et cela juste après avoir dévoilé, grâce à son point de vue omniscient, plusieurs de ses aventures : «- As-tu des nouvelles de Hope ? [...] J'ai secoué la tête. Non, aucune nouvelle de Hope » (Dickner 219) ou «Avez-vous reçu des nouvelles de Hope ? Elle me lança un regard interrogateur et rangea la bouteille sans la reboucher. - Moi ? Non. Pourquoi ? Je tripotais ma chope de bière, tout de même un peu craintif à me hasarder sur un terrain aussi intime » (238-239). Le savoir de ce narrateur est donc pour le moins contradictoire. L'ignorance est aussi le lot du narrateur de Dernier amour qui révèle sans hésitation son manque de savoir de certains détails ou faits : «L'un des deux pensa même. Je ne sais lequel. Que Paul avait bien de la chance d'avoir pour lui tout seul une femme comme elle »(Gailly 71). Les hésitations de ce narrateur ne permettent pas vraiment de conclure que l'on se trouve face à une omniscience régulière qui, comme nous l'avons vu avec Rabatel, peut comporter des variations de profondeur de perspective : «Il était dans sa chambre à l'étage. Non, il n'était pas dans sa chambre. À l'étage mais pas dans sa chambre. Il était dans la salle de bains »(Gailly 74). Ainsi, ce qui pouvait paraitre d'abord un problème de narration homodiégétique, devient aussi un problème de narration omnisciente. 


\section{Observation critique de la narration omnisciente}

Ces problèmes de l'omniscience renouvellent la réflexion sur les ambiguïtés d'un procédé largement employé en littérature comme convention de narration. L'omniscience permettait aux narrateurs des romans classiques de décrire le monde grâce à ce savoir illimité qu'elle leur assurait. Or, comment peut-on expliquer qu'un narrateur personnalisé puisse jouir de toutes ces connaissances? Comment est-il même possible de lire dans les pensées de tous les personnages ? Ce sont ces deux facultés singulières du narrateur qui se voient problématisées dans nos romans. Le fait d'attribuer l'omniscience à un narrateur homodiégétique ne nous semble donc pas fortuit mais un signe d'une réflexion contemporaine sur les règles de la narration, en particulier de la narration omnisciente. En effet, et pour résumer les propos de JonArild Olsen, dans les romans à la première personne, les transgressions des convenances reliées à la narration homodiégétique sont instantanément constatées par les lecteurs et les critiques. Ce n'est pas le cas dans les romans à la troisième personne où les transgressions de la vraisemblance pragmatique, quoique plus abondantes, n'y seraient que rarement relevées. Alors que le lecteur se demanderait nécessairement d'où le «je » a pu recueillir toutes les informations présentes dans son récit et s'attendrait à ce que celui-ci apporte des preuves à l'appui de ses dires, il admettrait de bon gré cette illusion cognitive dans les romans à la troisième personne. L'adhésion spontanée du lecteur au discours du narrateur hétérodiégétique reviendrait à l'appartenance de ce dernier à la même réalité fictive des événements qu'il rapporte. Ainsi, en accordant l'omniscience au narrateur homodiégétique, les romanciers contemporains chercheraient non seulement à étendre les frontières de la fiction jusqu'à la situation de narration homodiégétique, mais à rendre aussi plus manifestes les invraisemblances de l'omniscience qui souvent passent inaperçues pour les lecteurs.

En réalité, le point de vue du narrateur omniscient, même traditionnel, n'est pas forcément porteur de vérité. L'on voit souvent, par exemple, des narrateurs omniscients omettre des informations nécessaires pour la compréhension du déroulement des événements, et cela dans le but d'augmenter le suspense. Vitoux et Rabatel abordent ce point dans leurs différents articles et soutiennent la considération de tous les points de vue présents dans un texte. L'omniscience qui n'est pas incontestablement le véhicule du vrai est, par ailleurs, couramment limitée par l'espace et le temps. Le narrateur qui décrit une scène quelconque dans son récit, est contraint à limiter son savoir au lieu où se déroule cette scène et surtout à la durée de celle-ci : 
«L'omniscience, qu'il n'est pas question de rejeter, mais qu'il convient de mieux fonder, résulte moins de l'encyclopédisme du narrateur focalisateur [...] que du fait de savoir si les perceptions (et le savoir qu'elles véhiculent) sont limitées, ou non, à $\mathrm{S}^{\mathrm{o}}$ et $\mathrm{T}^{\mathrm{o}}[\ldots]$, limitées, ou non, par $\mathrm{S}^{\mathrm{o}}$ et $\mathrm{T}^{\mathbf{o}} \gg$ (Rabatel 100). De plus, l'omniscience est souvent reliée dans les romans au pouvoir d'action qu'est l'omnipotence. Mais cette suprématie présumée des narrateurs n'est qu'illusoire. Le narrateur raconte souvent des faits passés et n'a de ce fait aucun pouvoir pour changer le cours de l'histoire ou inverser les rôles des personnages puisque les faits se sont déjà produits dans le cadre, bien sûr, de la fiction. L'omnipotence présumée de la narration omnisciente est un artifice dont les limites sont assez clairement délimitées.

Ensuite, même si personne ne peut posséder un savoir illimité sur le monde, cela n'empêche pas néanmoins que le narrateur connaisse profondément l'histoire qu'il révèle et qu'il l'organise autant chronologiquement que thématiquement selon sa propre vision. Selon Marcel Vuillaume, le narrateur omniscient ressemble à un metteur en scène qui régit l'ensemble de l'œuvre, que ce soit les acteurs ou les scènes. Il ne serait donc pas ce démiurge qui décide du cours de l'histoire et des actes de ses personnages. Le déplacement de ce type de narrateur dans les différents points de vue intérieurs de ses personnages ou ce que Gérard Genette appelle aussi «focalisation zéro » permet d'illustrer ce caractère complexe de l'omniscience. En effet, afin de révéler la vie interne de chacun de ses personnages, le narrateur hétérodiégétique développe plusieurs scènes en «focalisation interne » tout en les disposant de manière à aboutir à une finale. Mais ce mouvement qu'effectue le narrateur d'un point de vue à l'autre, dissout finalement son propre point de vue et empêche le lecteur d'adhérer à une perspective déterminée qui l'oriente dans sa découverte des événements de l'histoire. En plus, dans ce type de narration, l'omniscience ne domine pas vraiment puisque la fréquente restriction du point de vue du narrateur au point de vue des personnages ne permet pas au premier de révéler beaucoup plus d'informations que n'en connaît le personnage. Comme le précise Rabatel à propos de la forte confusion entre focalisation zéro et focalisation interne variable : «Le passage d'une perspective illimitée à une perspective limitée n'est pas possible si la vision est celle du même référent, par le même focalisateur sauf si l'on change de focalisateur, ou si l'on change de destinataire : dès lors le problème ne se pose évidemment plus »(102-103). En adoptant le point de vue du personnage, le narrateur réduit nécessairement son savoir. L'omniscience n'est plus discernée et souvent ce type de narration se trouve confondu avec la narration homodiégétique variable. En somme, 
l'accès direct aux pensées des personnages minimise l'effet de l'omniscience. Par ailleurs, selon Percy Lubbock, la narration omnisciente, qu'il désigne par l'expression «narration panoramique », serait inférieure aux autres formes narratives du fait que la représentation du réel se voit ramenée au seul point de vue du narrateur, ce qui a pour conséquence de figer cette représentation. Pour Lubbock, alors que la variation des points de vue internes permet plus de diversité dans la manière de percevoir le monde, le point de vue unique du narrateur omniscient donnerait une image plate et mince de ce monde. C'est dire que le point de vue omniscient ne peut régner à lui seul dans un récit sous peine de dénaturer notre expérience du réel. Accompagné des autres points de vue, il lui est possible de fournir plus de richesse au récit. Le narrateur diversifie la profondeur de son savoir selon l'importance des scènes qu'il montre. C'est son habileté et son savoir-faire qui lui servent à rendre ce mouvement entre les différents points de vue internes inaperçu. L'omniscience comme technique narrative n'englobe pas seulement un savoir profond et infini du narrateur, elle fait appel aussi à l'habileté de ce dernier dans l'organisation de son histoire et surtout dans sa capacité d'agencer et de combiner les différents points de vue des personnages de son récit.

En conclusion, nos romans unissent la narration homodiégétique à la vision omnisciente. Cette union de deux types narratifs normalement incompatibles déstabilise la situation narrative dans les récits. Non seulement un narrateur homodiégétique ne peut généralement acquérir un savoir absolu sur le réel, mais le point de vue omniscient qu'il revendique dans nos romans n'assure pas une connaissance profonde du monde décrit. Son emploi ne fait que déstabiliser encore plus le réel représenté et affaiblir l'autorité des narrateurs. Même si le lecteur choisit d'adhérer aux discours de ces derniers, leur crédibilité tient à leur appartenance à la réalité de l'histoire qu'ils rapportent et non à une certaine foi en leurs connaissances.

\section{Bibliographie}

Dickner, Nicolas. Tarmac. Québec : Alto, 2009.

Gailly, Christian. Dernier amour. Paris : Minuit, 2004.

Genette, Gérard. Figure III. Paris : Seuil, 1983.

Lubbock, Percy. The Craft of Fiction. New York : Smith, 1947.

Olsen, Jon-Arild. L'esprit du roman : xuvre, fiction et récit. Berne : Lang, 2004.

Quiviger, Pascale. La maison des temps rompus. Montréal : Boréal, 2008. 
Rabatel, Alain. « De l'influence de la fréquence itérative sur l'accroissement de la profondeur de la perspective : un retour critique sur l'omniscience narratoriale et sur la restriction de champ du personnage ». Protée $28: 2$ (2000) : 93-104.

Vitoux, Pierre. «Focalisation, point de vue, perspective ». Protée 16 (1988) : 33-39.

Vuillaume, Marcel. Grammaire temporelle des récits. Paris : Minuit, 1990. 